\title{
The Mirror Module Design for the Cryogenic X-Ray Imaging Spectrometer on-board ORIGIN
}

\author{
Marco Barbera ${ }^{* a, b}$, Teresa Mineo ${ }^{c}$, Stefano Basso ${ }^{\mathrm{d}}$, Finn Erland Christensen ${ }^{\mathrm{e}}$, \\ Jan-Willem den Herder ${ }^{\mathrm{f}}$, Jelle Kaastra ${ }^{\mathrm{f}}$, Luigi Piro ${ }^{\mathrm{g}}$, Daniele Spiga ${ }^{\mathrm{d}}$, Coen van Baren ${ }^{\mathrm{f}}$ \\ ${ }^{a}$ Dipartimento Di Fisica, Università di Palermo, Via Archirafi 36, 90123 Palermo, Italy \\ ${ }^{b}$ INAF-Osservatorio Astronomico G.S. Vaiana, Piazza del Parlamento 1, 90134 Palermo, Italy \\ ${ }^{\mathrm{c}}$ INAF-Istituto di Astrofisica Spaziale e Fisica Cosmica, Via U. La Malfa 153, 90146 Palermo, Italy \\ ${ }^{\mathrm{d} I N A F-O s s e r v a t o r i o ~ A s t r o n o m i c o ~ d i ~ B r e r a, ~ V i a ~ B i a n c h i ~ 46, ~} 23807$ Merate (Lc), Italy \\ ${ }^{\mathrm{E}}$ Danish National Space Center, Juliane maries Vej 30, 2100 Copenhagen, Denmark \\ ${ }_{\text {f }}$ SRON Netherlands Institute for Space Research, Sorbonnelaan 2, 3584 CA Utrecht, the Netherlands \\ ${ }^{\mathrm{g}}$ INAF-Ist. di Astrofisica Spaziale e Fisica Cosmica, Via Fosso del Cavaliere 100, 00133 Roma, Italy
}

\begin{abstract}
ORIGIN is a medium size high-energy mission concept submitted to ESA in response to the Cosmic Vision call issued on July 2010. The mission will investigate the evolution of the Universe by performing soft X-ray high resolution spectroscopic measurements of metals formed in different astrophysical environments, from the first population III stars at $\mathrm{z}>7$ to the present large scale structures. The main instrument on-board ORIGIN will be a large format array of TES X-ray micro-calorimeters covering a FOV of 30' at the focal plane of a grazing incidence optical module with a focal length of $2.5 \mathrm{~m}$ and an angular resolution of 30" $\mathrm{HEW}$ at $1 \mathrm{keV}$. We present the optical module design which is based on hybrid technologies, namely Silicon Pore Optics for the outer section and Ni electro-forming for the inner section, and we present the expected performances based on test measurements and ray-tracing simulations.
\end{abstract}

Keywords: X-ray optics, X-ray Astronomy, Space missions.

\section{INTRODUCTION}

ORIGIN is a high-energy astrophysics mission designed to study the metal creation from cosmic dawn, which has been proposed in response to the M3 mission call of ESA issued in July 2010. Using high-spectral resolution in the soft X-ray band, ORIGIN will be able to identify the physical conditions of all abundant elements between $\mathrm{C}$ and $\mathrm{Ni}$ over a red-shift range up to $z=10$ and beyond. The mission will answer questions including: When were the first metals created? How does the cosmic metal content evolve? Where do most of the metals reside in the Universe? What is the role of metals in structure formation and evolution? To reach out the early Universe, ORIGIN will use Gamma-Ray Bursts (GRB) to study the local environment of the host galaxies. This requires the capability to slew the satellite in less than a minute to the GRB location. Fast re-pointing is achieved by a dedicated Controlled Momentum Gyro, and a low background is obtained selecting a low Earth orbit. The ORIGIN mission includes three science instruments:

- TED (Transient Event Detector): a coded mask hard X-ray detector (5-200 keV) to identify and localize 400 GRB's per year. A unique feature of TED is its wide field coverage $(4 \mathrm{sr})$ resulting in a wealth of additional science for transient events. TED will have the sensitivity to detect $\sim 65$ GRB's $(z>7)$ over a 5 year mission.

* marco.barbera@unipa.it; phone+39091 7571113; fax +39091 7571129

EUV and X-Ray Optics: Synergy between Laboratory and Space II, edited by René Hudec, Ladislav Pina, Proc. of SPIE Vol. 8076, 80760A · C 2011 SPIE · CCC code: 0277-786X/11/\$18 · doi: 10.1117/12.889269 
- CRIS (Cryogenic X-Ray Imaging Spectrometer): a high spectral resolution $(<2.5 \mathrm{eV}) \mathrm{X}$-ray telescope with a TES micro-calorimeter array $(0.2-8 \mathrm{keV})$, with a large collecting area to enable element fingerprinting, and with a low background to study clusters and map a region of $2 \times 2.5 \mathrm{deg}^{2}$ to determine the physical properties of the WHIM.

- BIRT (Burst InfraRed Telescope): an infrared telescope with a spectral resolution of 20 to 1000 over the range of $0.5-1.7 \mu \mathrm{m}$, to determine GRB red_shifts in case of low metallicities $(\mathrm{z}=5.5-12$ with $\mathrm{HAB}=22)$, perform complementary measurements of the chemical abundances of low ionization species and probe the presence of dust.

The Cryogenic Imaging Spectrometer (CRIS) is the prime instrument of the mission. It allows wide field imaging of a $30^{\prime}$ FOV area on the sky with an effective area $>1500 \mathrm{~cm}^{2}$ at $1 \mathrm{keV}$ and $>150 \mathrm{~cm}^{2}$ at $6 \mathrm{keV}$. The detector has three different sections including an inner array of $26 \times 26$ pixels with energy resolution of $2.5 \mathrm{eV}$, an outer array of $72 \times 72$ pixels with energy resolution $<5 \mathrm{eV}$, and a third array $20 \times 20$ pixels located at on one side of the outer array for detecting GRBs. In the outer array four pixels are read out by a single TES connected by 4 different strong thermal links. This type of detector allows the identification of the X-ray absorbing pixel from the pulse shape before the TES and the 4 absorbers come into thermal equilibrium [1]. The GRB detector array is placed $8 \mathrm{~mm}$ out of focus to spread the Point Spread Function (PSF) over a sufficiently large number of pixels so that the maximum count-rate capability of each pixel is not exceeded when observing bright GRB afterglows. Figure 1 shows the layout of the detector at the focal plane of the CRIS optical module. Its confusion limit is $10^{-15} \mathrm{erg} \mathrm{cm}^{-2} \mathrm{~s}^{-1}$ for $0.5-2 \mathrm{keV}$ and its point source line detection sensitivity at $0.5 \mathrm{keV}(5 \sigma)$ is typical $210^{-7}$ photons $\mathrm{cm}^{-2} \mathrm{~s}^{-1}$ for a $100 \mathrm{ks}$ observation.

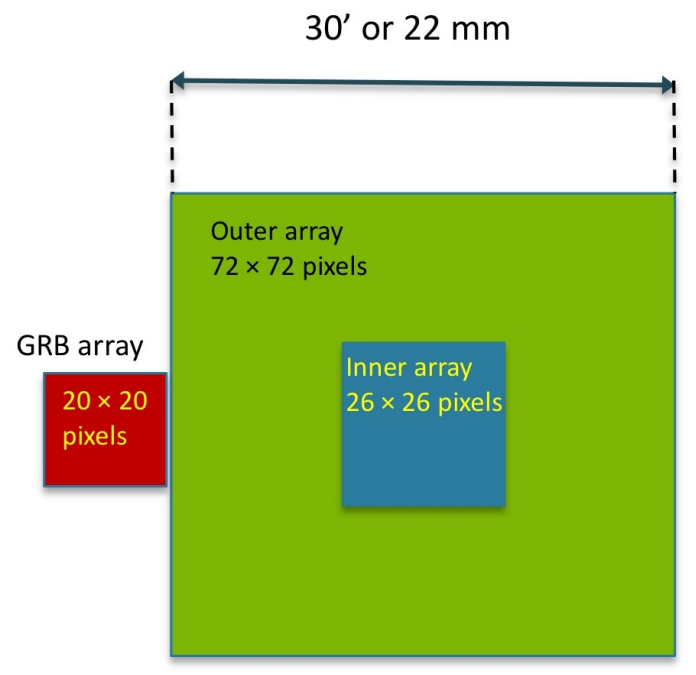

Figure 1. Layout of the TES micro-calorimeter detector at the focal plane of the CRIS optical module. The GRB detector is located $8 \mathrm{~mm}$ out of focus to spread the PSF of bright sources over a larger number of pixels.

The CRIS instrument is largely based on the most significant technology developments carried out for the International X-ray Observatory (IXO) and for ASTRO-H. In particular, the optics development will rely partially on the Silicon Pore Optics (SPO) technology under development for IXO [2][3] and partially on the Ni electroforming optics technology already proven in several X-ray telescopes[4][5][6][7]. The instrument design has been optimized to give a high grasp (the effective area on-axis times the solid angle of the field of view) by having a short focal length $(2.5 \mathrm{~m})$. Considering the size of the detector, the useful field of view (FOV) of the optical module is 30'. With this configuration we maximize the effective area at lower energies $(<1 \mathrm{keV})$ while retaining a reasonable effective area at $6 \mathrm{keV}\left(>150 \mathrm{~cm}^{2}\right)$. The short focal length also implies a low moment of inertia, necessary for fast re-pointing.

\section{THE OPTICAL MODULE OF THE CRYOGENIC IMAGING SPECTROMETER}

To meet the ORIGIN requirements for the CRIS instrument of high effective area at $1 \mathrm{keV}$, a working range of $0.2-8$ $\mathrm{keV}$, and an angular resolution $<30$ " HEW on-axis we have proposed an optical module design based on a hybrid mirror 
technology: the outer part of the mirror will be built with SPO and the inner part with the standard Ni electroforming technology (Figure 2). This choice reduces the mass since SPO are very lightweight. For the high-energy response the inner mirror is needed since the feasibility of SPO optics has only been demonstrated at radii down to $0.3 \mathrm{~m}$. The optical module will be a conical approximation to Wolter-I geometry.

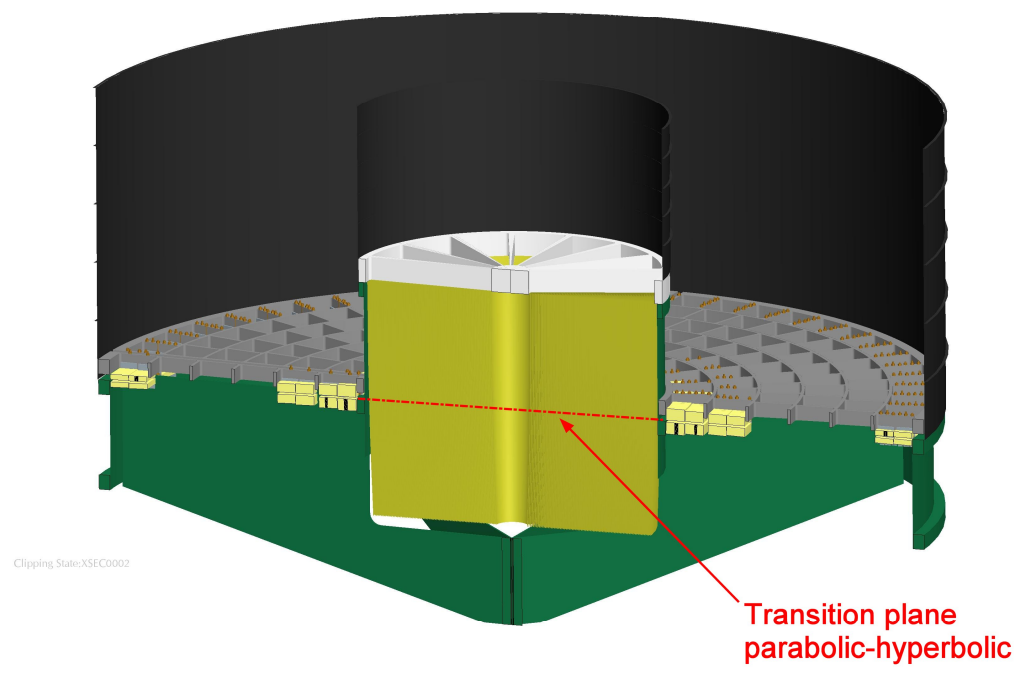

Figure 2. Design of the CRIS hybrid mirror. Inner part: classical Ni formed shells mounted on a spider. Outer part: SPO modules (yellow) mounted in four petals (grey). The green part is the structural support.

In the following sections we describe the main characteristic of the proposed technologies and the design parameters of the outer (SPO) and inner (Ni electroforming) mirrors.

\subsection{Outer Section - Silicon Pore Optics}

SPO relies on using the optically-polished surface of coated Si wafers as reflectors and properly stacking a set of these wafers in elements that, placed behind each other, can approximate the required geometry of a double reflection focusing mirror module[7][8]. A large number of this elements named mirror modules (MM), each one with proper geometry for a given radius can be mounted together to fill the entrance pupil of the optical module and provide large collecting area. The construction process of an SPO stack follows these main steps [9][10]:

1. Procurement of a high surface quality Si wafer;

2. Growth of an oxide layer to protect the optical surface during etching processes;

3. Dicing to make square plates;

4. Ribbing the plates by dicing (sawing) to create pores, then wedging to determine proper grazing angle inclination of the plate;

5. Coating the optical surface with a metal to increase X-ray reflectivity leaving uncoated channels where ribs of an adjacent plate will be bonded in the stacking process;

6. Bending plates and stacking a few tens of them onto a concave mandrel, namely cylinder, cone or Wolter-I depending on the required angular resolution. E.g. for a focal length of about $20 \mathrm{~m}$ like IXO, a cylindrical mandrel would be sufficient for 30" Half Energy Width (HEW) resolution, conical for 10" and true Wolter-I is needed for $<5$ ". For the CRIS optics of ORIGIN conical mandrel can be used to bend the plates;

7. Optical bonding and annealing to rigidly attach plates while they are hold curved onto the mandrel. The annealing is still an option, it may be replaced by Plasma Activation applied before stacking a plate;

8. Release of the concave tool that hold the plates onto the mandrel;

9. Mounting and integration 
When a plate is bent the optical surface faces the center of curvature of the concave mandrel while ribs are facing down and are thus forced to slightly open to point to a common centre. This effect is not negligible for the inner radii of the optical module. For example bending of a plate onto a concave mandrel with $30 \mathrm{~cm}$ curvature radius causes in each pore the ribs to open on top by $2.6 \mu \mathrm{m}$. Since this effect is cumulative, in the outer pore of a plate ( 25 pores off the center), the top edge of the ribs will be off by $65 \mu \mathrm{m}$ with respect to its position in a flat plate. This effect could cause the outer ribs to partially get out of the open channels of the next plate in the stacking process and overlap the metal coated reflecting surface thus implying a non-ideal bonding. To prevent this, the metal coating of the optical surface, which leaves open channels for bonding the next plate in the stack, will be performed using a different lithographic mask every small number (TBD) of plates. The masks will all have a constant spacing of the open channels that changes to follow the opening of the ribs as the curvature radius changes from the inner radii to the outer radii of the optic (Cosine Research (NL) private communication).

A fully automated stacking robot is operating to assemble stacks of plates according to the IXO optics design with $20 \mathrm{~m}$ focal length and a bending radius of $0.74 \mathrm{~m}$ [11][12]. For our application we have to stack shorter wafers with an inner bending radius of $0.3 \mathrm{~m}$. The smaller bending radius has been studied by finite element modeling and has been demonstrated by bending and bonding of two mirror plates with this radius (Cosine Research (NL) private communication).

An angular resolution (HEW) $<20$ " has been repeatedly demonstrated by X-ray pencil beam measurements performed at PTB in Berlin for a set up to 45 plates of an IXO mirror module, mounted in representative flight configuration [10][13]. These results indicate that in the case of ORIGIN, the 30" HEW requirement on-axis, being dominated by the conical approximation to the Wolter-I optics, is achievable.

To increase the mirror reflectivity, the SPO will be coated with a three layer reflecting surface: C (25 $)-\mathrm{Ni}(25 \AA)-\mathrm{Pt}$ (300 ̊). To estimate the effective area we assumed the proven thickness of the reflectors $(170 \mu \mathrm{m})$ but FEM calculations suggest that we can reduce this to $120 \mu \mathrm{m}$ resulting in a gain of $10 \%$ of the area. For the design of the SPO covering the outer part of the CRIS optical module we built upon the experience matured for the IXO optical module [14][15]. The coverage of the entrance pupil will be obtained by assembling a large number of mirror modules (MM) each one containing the $1 \alpha$ and $3 \alpha$ stacks of plates. Each stack contains 40 plates with a thickness of $0.17 \mathrm{~mm}$, and spacing between plates equal to $0.775 \mathrm{~mm}$. The pore width is $1 \mathrm{~mm}$, and the rib width is $0.17 \mathrm{~mm}$.

Figure 3 shows the layout of one SPO quadrant covering the outer part of the CRIS optical module. The quadrant has been divided in six annuli, each one containing two rows of MM. The separation between MM in a row is $10 \mathrm{~mm}$, while the separation between MM in two adjacent annuli is $15 \mathrm{~mm}$.

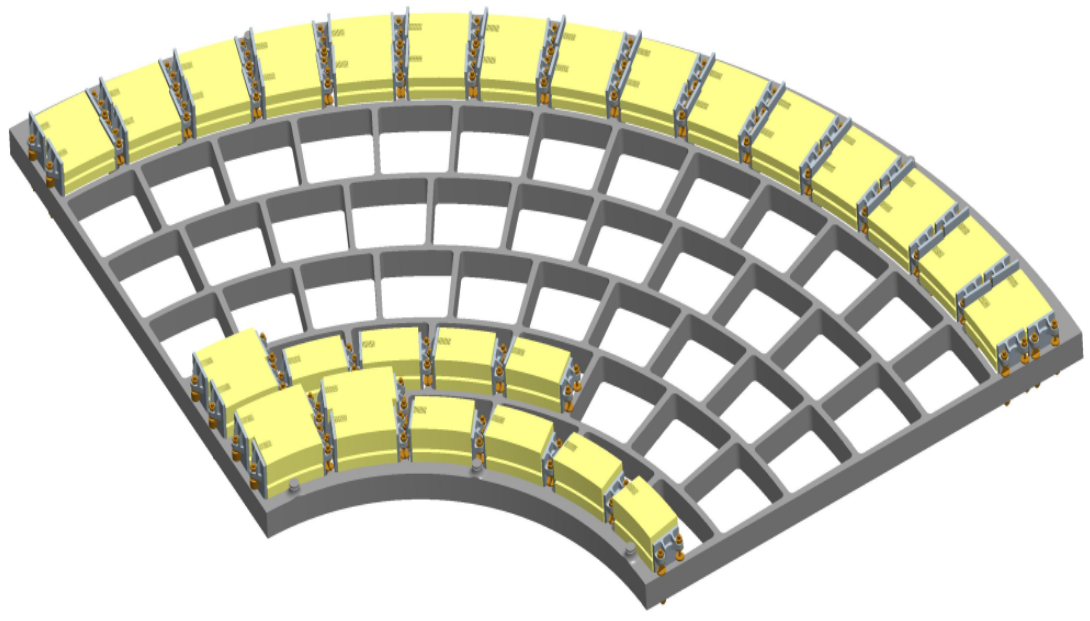

Figure 3. Layout of one quadrant of the SPO covering the outer part of the CRIS optical module.

The stack width varies from $51.4 \mathrm{~mm}$ to $63.6 \mathrm{~mm}$, going from the inner to the outer radii, which is a bit less than the 65.7 $\mathrm{mm}$ currently under development by Cosine Research (NL). This difference should not be critical, however, the arrangement of the mounting structure and blocking areas can be optimized to obtain stack widths closer to the currently produced width of $65.7 \mathrm{~mm}$. Figure 3 does not show the spider support structure ( 8 spokes over the full optical module 
area). Since the spacing of the reflecting surface of the plates is constant by construction and equal to $0.775 \mathrm{~mm}$, in order to maintain a zero vignetting on axis the height of the plates $(\mathrm{P}+\mathrm{H})$ changes along the radii from $45.0 \mathrm{~mm}$ for the inner radius to $17.3 \mathrm{~mm}$ for the outer radius. The same condition is obtained for the inner $\mathrm{Ni}$ electroforming optics by maintaining a constant length of $400 \mathrm{~mm}$ and changing the spacing between shells from $0.72 \mathrm{~mm}$ for the inner radius to $4.7 \mathrm{~mm}$ for the outer radius. Table 1 summarizes some of the main design parameters of each one of the four SPO quadrants.

Table 1. Design parameters of one quadrant of the outer SPO section of the CRIS optical module.

\begin{tabular}{|c|c|c|c|c|c|}
\hline Row & $\begin{array}{c}\text { Rmin }\left(^{*}\right) \\
{[\mathrm{mm}]}\end{array}$ & $\begin{array}{c}\text { Rmax }(*) \\
{[\mathrm{mm}]}\end{array}$ & $\begin{array}{c}\text { Number of MM } \\
\text { per row }\end{array}$ & $\begin{array}{c}\text { Stack width } \\
{[\mathrm{mm}]}\end{array}$ & $\begin{array}{c}\text { Stack height } \\
{[\mathrm{mm}]}\end{array}$ \\
\hline 1 & 270.000 & 300.225 & 6 & 54.9 & 42.6 \\
\hline 2 & 303.225 & 333.450 & 6 & 63.6 & 38.0 \\
\hline 3 & 343.450 & 373.675 & 8 & 51.8 & 33.6 \\
\hline 4 & 376.675 & 406.900 & 8 & 58.3 & 30.9 \\
\hline 5 & 416.900 & 447.125 & 10 & 50.0 & 28.0 \\
\hline 6 & 450.125 & 480.350 & 10 & 55.2 & 26.0 \\
\hline 7 & 490.350 & 520.575 & 11 & 54.6 & 23.4 \\
\hline 8 & 523.575 & 553.800 & 11 & 59.3 & 22.4 \\
\hline 9 & 563.800 & 594.025 & 13 & 52.7 & 20.8 \\
\hline 10 & 597.025 & 627.250 & 13 & 56.8 & 19.8 \\
\hline 11 & 637.250 & 667.475 & 15 & 51.4 & 18.6 \\
\hline 12 & 670.475 & 700.700 & 15 & 54.9 & 17.6 \\
\hline
\end{tabular}

(*) At plane between Parabola and Hyperbole

\subsection{Inner Section - Ni electroforming}

The inner section of the CRIS optical module is based on mirrors replicated from mandrels, obtained using the Nickel electroforming technique. This technology is in use since decades[16] to manufacture grazing incidence X-ray mirrors of any profile (double cone, Wolter-I, or polynomials) with a good angular resolution $(\sim 15 \operatorname{arcsec})$ while keeping the thickness of the mirrors low (a few tenth $\mathrm{mm}$ ), therefore enabling a dense nesting of mirrors. The Ni electroforming has already been used successfully to manufacture the mirrors of Beppo-SAX[16], Newton-XMM[17], SWIFT-XRT[18] with single Gold layer (TRL 9), and is also one of the technologies in use for hard X-ray optics with multilayer coatings[19][20][21]. The manufacturing technique consists of a figured mandrel in Aluminum with an amorphous Nickel-Phosphorous coating, with the negative profile of the mirror to be produced. The chemical electroless Nickel is a suitable material to superpolish the mandrel surface down to a few angstrom rms level, an important requirement for the replicated mirror to keep the X-ray scattering as low as possible. A thin film (50-150 nm) of Gold [22] is then evaporated on the mandrel, and the Nickel mirror walls are grown on the Gold layer in an electrochemical bath. The mirror is finally released by cooling the mandrel, owing to the much higher CTE of the Aluminum mandrel with respect to the Nickel mirror. In this process, the Gold layer acts both as a soft X-ray reflecting layer, and as a release agent since its adhesion to the electrochemical Nickel of the mandrel is very low. A particular attention has to be paid to the optimization of the Gold layer thickness[22], that has to be thick enough to chemically isolate the mirror Nickel from the Nickel coating of the mandrel, but at the same time thin enough to limit the growth of the roughness. A multilayer coating can be finally deposited on the inner side of the mirror shell by means of two linear sources[23]. The mandrel, after a thorough cleaning, can be reused to produce another mirror of the same profile and size.

A critical aspect of the electroforming process is the accuracy of profile replication: the electroforming and release process introduce an internal stress in the texture of the Nickel mirror, which may deform the mirror if its rigidity is not sufficient. This risk is present in particular when the mirrors have a very low incidence angle to enhance the reflectivity in hard X-rays, because a very large number of mirrors have to be employed to fill the aperture of the module. In this case, the mirrors walls must be very thin; otherwise, the effective area/mass ratio of the optics becomes unfavorable. Two methods can be used to improve the imaging quality:

1) The adoption of galvanic alloys with a higher rigidity than pure Nickel, like the Nickel (80\%) - Cobalt (20\%) alloy (NiCo). The adoption of the NiCo alloy for manufacturing Wolter-I has been developed at the NASA-Marshall Space Flight Center [24] and extensively studied at Harvard - Center for Astrophysics (Boston, USA) as a candidate for the focusing mirrors of the Constellation-X hard X-ray telescope[24][25]. In the last years, INAF/Osservatorio Astronomico di Brera and Media-Lario technologies have tested the adoption of the NiCo alloy 
for the demonstrators of the SIMBOL-X[26][27] and NHXM[28] hard X-ray telescopes. The NiCo technology (TRL 5) is not as advanced as pure Nickel, but it is very promising since it exhibits a higher elastic limit than pure Nickel[23] therefore it can better outstand the stress that arises during the electroforming, the release, and the subsequent handling and integration. In addition, the polycrystalline micro-texture of the NiCo alloy is much finer than pure $\mathrm{Ni}$, resulting in a shining color and a better micro-roughness of the optical surface[23][25].

2) The optimization of the mirror walls thickness. An excessive thickness would increase the overall mass and decrease the clear aperture of the mirror with a consequent decrease of effective area. On the other side, the required imaging quality of 30 arcsec HEW is mostly determined by the figure accuracy of the mirrors, which can be degraded easily if the mirrors are too thin and prone to deform. The angular resolution turns out to be a function of the ratio thickness/radius, $k$, assumed to be constant throughout the mirror module: more exactly, the analytical relation between $k$ and the HEW[29] is expressed by a power-law, HEW $\approx H_{0} k^{-z}$, with $z=0.566$, and $H_{0} \approx 0.548$ arcsec. These coefficient values are, strictly speaking, referred to the NHXM case of $600 \mathrm{~mm}$-long mirror shells. For shorter mirror shells like ORIGIN's, the optical quality is expectedly more affected by the edge effects, but for a preliminary analysis the same values can be assumed. Making different hypotheses on the required angular resolution, we derive the results presented in Table 1 . We note that the study of the effective area has so far assumed the thickness of mirrors to take on two values: $0.3 \mathrm{~mm}$ for the 43 outermost mirrors and $0.2 \mathrm{~mm}$ for the 43 innermost ones, with a total mirror mass of $58 \mathrm{~kg}$, without accounting for the spiders and the other supporting structures. These values of thickness are in the range of the solution No. 2 in Table 2, i.e., the one yielding a 20 arcsec HEW. Improving further the angular resolution by $5 \operatorname{arcsec}$ (No. 3) would require an increase of the total module mass by almost a factor two.

Table 2: Optimal shell thickness of the CRIS inner mirror module as a function of predicted angular resolution (HEW). As per the current optical design, the maximum radius is $246 \mathrm{~mm}$ and the minimum radius is $34.7 \mathrm{~mm}$. A lower limit of $0.1 \mathrm{~mm}$ for the thickness has been assumed, with a negligible impact on the total mass.

\begin{tabular}{|l|l|l|l|l|}
\hline $\begin{array}{l}\text { HEW } \\
(\operatorname{arcsec})\end{array}$ & $\begin{array}{l}\text { Thickness/radius } \\
\text { ratio, } k\end{array}$ & $\begin{array}{l}\text { Max. thickness } \\
(\mathrm{mm})\end{array}$ & $\begin{array}{l}\text { Min. thickness } \\
(\mathrm{mm})\end{array}$ & $\begin{array}{l}\text { Total module } \\
\text { mass }(\mathrm{kg})\end{array}$ \\
\hline 30 & 0.00085 & 0.21 & 0.1 & 29 \\
\hline 20 & 0.00174 & 0.43 & 0.1 & 54 \\
\hline 15 & 0.00290 & 0.71 & 0.1 & 90 \\
\hline
\end{tabular}

\section{PERFORMANCE SIMULATION}

The ray-tracing used to check the performance of the designed optical module is a modified version of the code already used to calibrate Beppo_SAX[30] and SWIFT-XRT optics[31], to evaluate the performance and to design the baffle for reducing the stray-light at the focal plane for the telescopes foreseen in EDGE[32] and SIMBOL-X [33]; moreover, a modified version of the code has also been used to simulate reflection from spiral optics[34][35].

It is a stand-alone software that follows, from the pupil to the focal plane, photons that are reflected from the mirror surface according to geometrical optics and mirror reflectivity computed using the standard routines based on solution of the Fresnell equations. Effects of the surface micro-roughness can be introduced either with a phenomenological model that takes into account the scattering dependence on energy, or with a Gaussian distribution whose sigma, independent from energy, is properly tuned to reproduce the PSF characteristics. The code is able to compute the reflectivity of grazing incident photons from several coating of the reflecting surface ( $\mathrm{Au}, \mathrm{Ni}, \mathrm{Pt}$, Ir, and multilayers) using tables, theoretical formulas and experimental results. It is moreover able to model the interaction of protons with the optics[36] that, according to Chandra and XMM experience[37], acts as focusing system also for these charged particles increasing the instrumental background at the focal plane.

The SPO have been modeled as nested shells with a conical approximation taking into account the effective area reduction due to the mounting structure of the mirror according to the mechanical design of the optic shown in Figure 2. Pores are simulated with a rectangular shape of $1 \mathrm{~mm}$ width (reflecting side) and $0.605 \mathrm{~mm}$ height. The separation between pores is $0.170 \mathrm{~mm}$ (ribs), which is the same as the thickness of the silicon plates. Effects of the distortion of the plates from the pure conical shape and mounting tolerances of the stacks are included, adding a phenomenological description of the scattering, with a Gaussian distortion from the pure geometrical reflection with an HEW of 16". The adopted value is based on the current best results obtained from imaging tests performed on a double reflection MM with 45 plates[38]. 
The Ni electroformed shells of the inner optical module have been modeled with a Wolter-I geometry (Parabola + Hyperbole) assuming that the spider support structure covers $10 \%$ of the entrance pupil geometric area. Also in this case we have introduced a Gaussian distortion from the nominal geometrical reflection with an HEW of 12" to account for figure errors, micro-roughness and mounting tolerances. The adopted value is based on the experience from the calibration of the SWIFT-XRT telescope[31]. Table 3 summarizes the main parameters of the two modeled optical modules.

Table 3: Main parameters of the inner and outer sections of the CRIS optical module

\begin{tabular}{|l|l|l|}
\hline & \multicolumn{1}{|c|}{ Outer Module } & \multicolumn{1}{|c|}{ Inner Module } \\
\hline Technology & Silicon Pore Optics & Ni Electroforming \\
\hline Geometry & Conical Approximation & Wolter-I \\
\hline Reflecting Coating & C-Ni-Pt on Si & $\begin{array}{l}\text { Au (baseline) or } \\
\text { Pt-C multilayer }\end{array}$ \\
\hline Focal Length [cm] & 250 & 250 \\
\hline R_min [cm] & 27.05 & 3.54 \\
\hline R_max [cm] & 67.05 & 25.07 \\
\hline Height_min (P+H) [cm] & 1.73 & 40 \\
\hline Height_max $(\mathrm{P}+\mathrm{H})[\mathrm{cm}]$ & 4.5 & 40 \\
\hline Grazing Angle_min [deg] & 1.54 & 0.20 \\
\hline Grazing Angle_max [deg] & 3.75 & 1.43 \\
\hline Geometric Area [cm $\left.{ }^{2}\right]$ & 12450 & 1960 \\
\hline Area covered by Support Structures & $31 \%$ & $10 \%$ \\
\hline Area covered by plate/shell thickness & $22 \%$ & $8 \%$ \\
\hline Total Useful Reflecting Area [cm $\left.{ }^{2}\right]$ & 5850 & 1590 \\
\hline N. of Plates/Shells & 480 & 86 \\
\hline Plates/Shell Thickness [mm] & 0.17 & 0.2 [inner 43 shells] \\
& & 0.3 [outer 43 shells] \\
\hline Mass of Plates/Shells [Kg] & 26 & 58 \\
\hline Mass of Supporting Structure [Kg] & $65\left(^{*}\right)$ & 25 \\
\hline
\end{tabular}

(*) Including the carbon fiber structure $(22 \mathrm{Kg})$ depicted in green color in figure 2.

\subsection{Effective Area}

The effective area (EA) on-axis as a function of the incident photon energy has been derived using the ray-tracing code separately for the inner and outer mirrors. For the inner mirror built with Ni electroforming technology we have modeled two different reflecting coatings. A pure Au coating applied to all reflecting shells (baseline for the mission) and a Pt-C multilayer (ML) coating applied to the outer shells to be considered as a case study and back-up solution. For the ML case the mirror shells have been divided in 6 groups: shells with incident angle $<0.5$ deg belonging to group 1 have been coated with $\mathrm{Au}$, the shells of the other five groups have been coated with a ML recipe with a constant $\mathrm{D}$ spacing for each shell but variable with the shell radius as reported in Table 4. For all ML recipes we have used a $\Gamma=0.6(\mathrm{C}$ thickness $/ \mathrm{Pt}$ thickness), and a number of bi-layers in the ML stack $=15$.

Table 4: D spacing values of the ML recipe for five groups of shells.

\begin{tabular}{|c|c|c|}
\hline ML Recipe & D spacing $[\AA]$ & Incident Angles [deg] \\
\hline 1 & 100 & $0.5-0.7$ \\
\hline 2 & 82 & $0.7-0.9$ \\
\hline 3 & 65 & $0.9-1.1$ \\
\hline 4 & 52 & $1.1-1.3$ \\
\hline 5 & 45 & $1.3-1.5$ \\
\hline
\end{tabular}


Figure 4 shows the plots of the calculated effective area for the inner optical module with Au coating (left panel) and with the ML coating on the right panel. The ML recipe has been designed to provide an increased EA at $6.5 \mathrm{keV}$. The EA at $6.5 \mathrm{keV}$ is $300 \mathrm{~cm}^{2}$ with the ML and $159 \mathrm{~cm}^{2}$ with a bare Au coating.
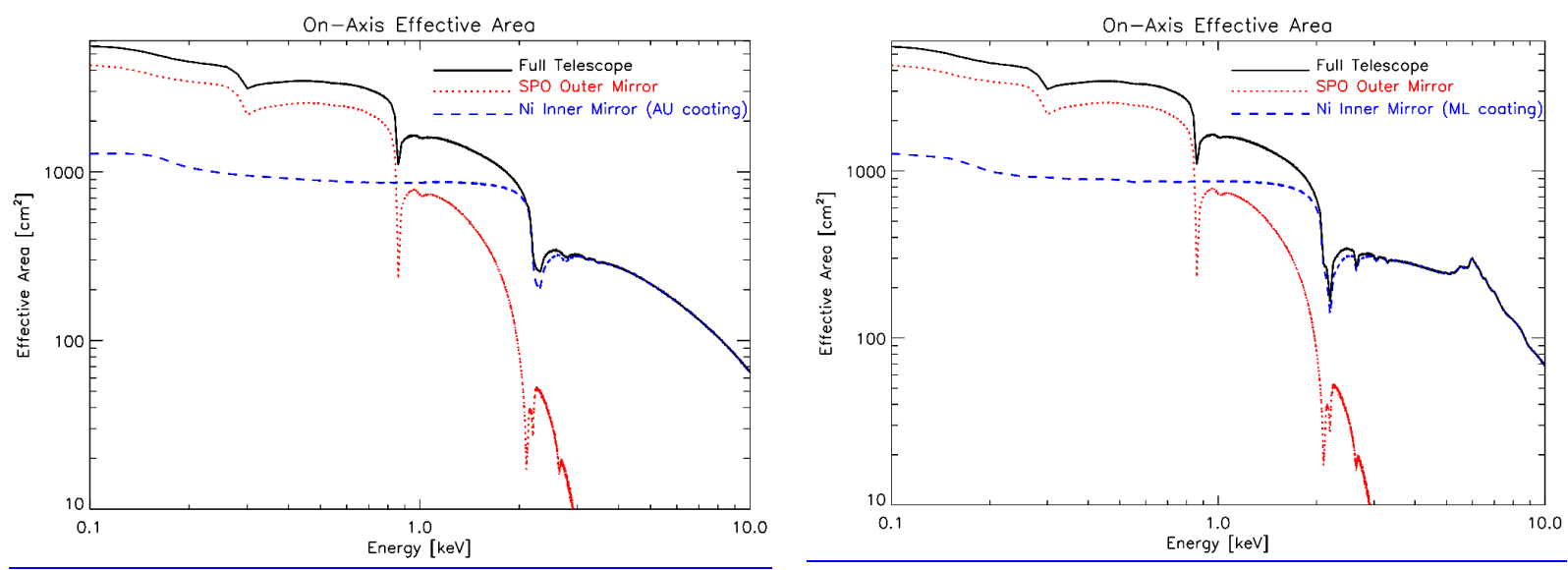

Figure 4. Effective Area vs. incident photon energy for the inner mirror module (dashed blu line), the outer mirror module (dotted red line), and the entire mirror module (black solid line). The left panel shows the EA calculated with bare Au coating onto the inner mirror shells, while the right panel with ML coating.

\subsection{Angular Resolution}

The scientific requirement of an angular resolution of 30" HEW on-axis can be achieved using a conical approximation to the Wolter-I geometry for the outer mirror module, where the replication of Wolter-I mandrels has not yet been fully developed although it has been considered within the IXO development program[10]. The inner mirror module has been designed according to the Wolter-I geometry since its implementation with the Ni electroforming technology is mature and has already been proven in space. Figure 5 shows the HEW angular resolution vs. off-axis angles at three different photon energies, namely $0.5,1.5$ and $6.5 \mathrm{keV}$.
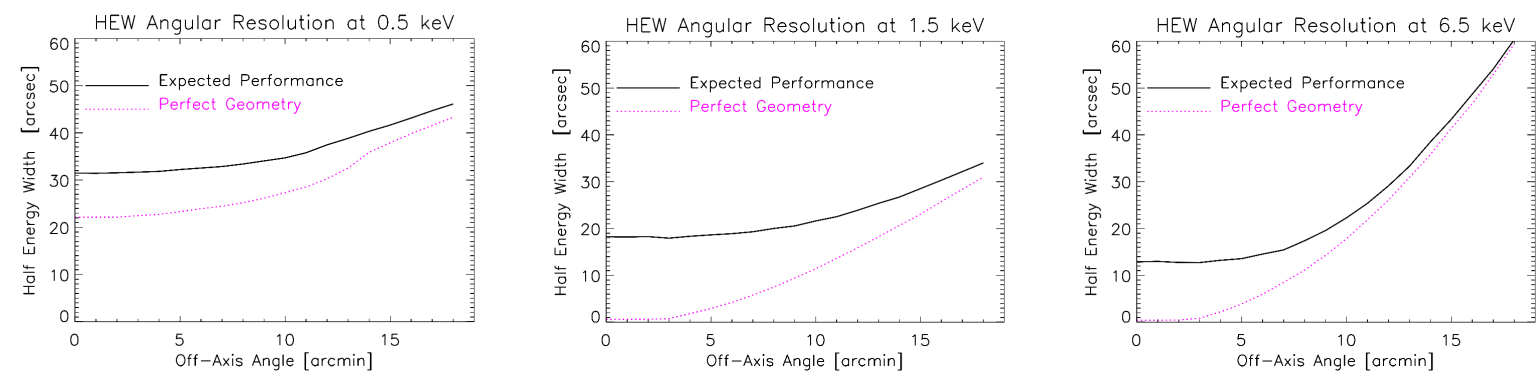

Figure 5. Angular resolution HEW vs. off-axis angle at three different energies, namely $0.5,1.5$, and $6.5 \mathrm{keV}$ from left to right.

Figure 6 shows images of the PSF at the same energies both on-axis and at three different off-axis angles, namely 5,10 , and 15 arcmin. At $0.5 \mathrm{keV}$ the EA and thus the angular resolution performance is largely dominated by the outer mirror module. The on-axis angular resolution is marginally above the scientific requirements, and only slowly deteriorates with increasing off-axis angle due to the short length of the mirror, which minimizes the spherical aberration. At $6.5 \mathrm{keV}$, only the inner mirror module contributes to the EA and the angular resolution is thus dominated by the Wolter-I geometry. The angular resolution on-axis is very good but it deteriorates faster at increasing off-axis angles due to the larger length of the mirror and thus non-negligible spherical aberration. A slight Coma aberration can be noticed in 
Figure 6 for the PSF images at low energies and large off-axis angles. At $6.5 \mathrm{keV}$, where only the inner mirror module contributes with the smallest incidence angles, the PSF images at large off-axis are strongly affected by vignetting.

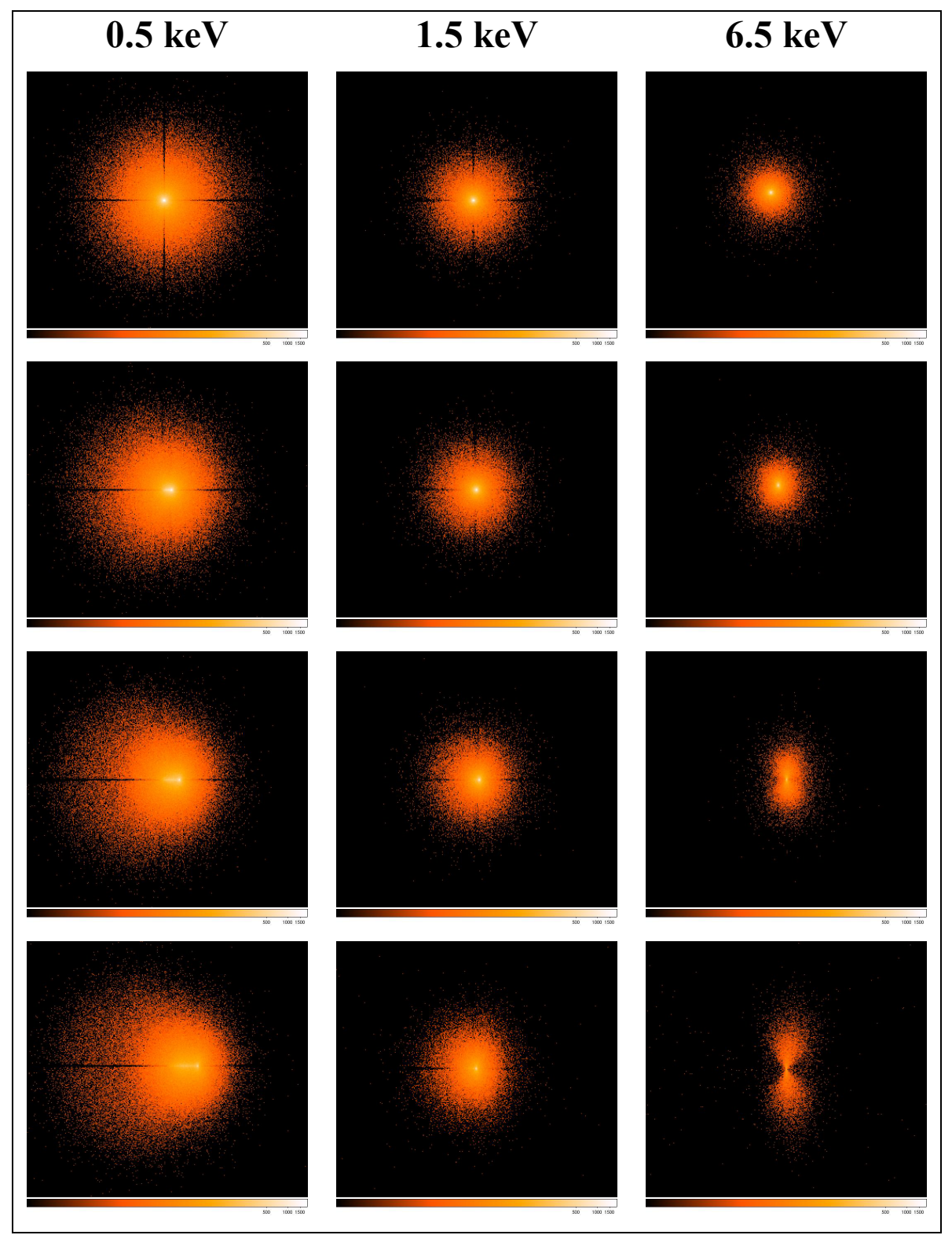

Figure 6. Images of the PSF at $0.5,1.5$, and $6.5 \mathrm{keV}$ incident photon energies, on-axis (first row), and 5, 10, and 15 arcmin off-axis (row 2, 3 and 4). Each image is $4 \times 4 \operatorname{arcmin}^{2}$.

\subsection{Vignetting}

Figure 7 shows the vignetting of the full optical module vs. off-axis angle at three different incident photon energies, namely $0.5,1.5$, and $6.5 \mathrm{keV}$. At low energies, where the EA is dominated by the outer mirror, the vignetting is not severe even at the edge of the FOV of the micro-calorimeter TES array. At high energies, where only the inner mirror provides EA, the vignetting is quite significant since the off-axis angle becomes comparable with the angles of incidence onto the inner shells of the inner mirror module. 

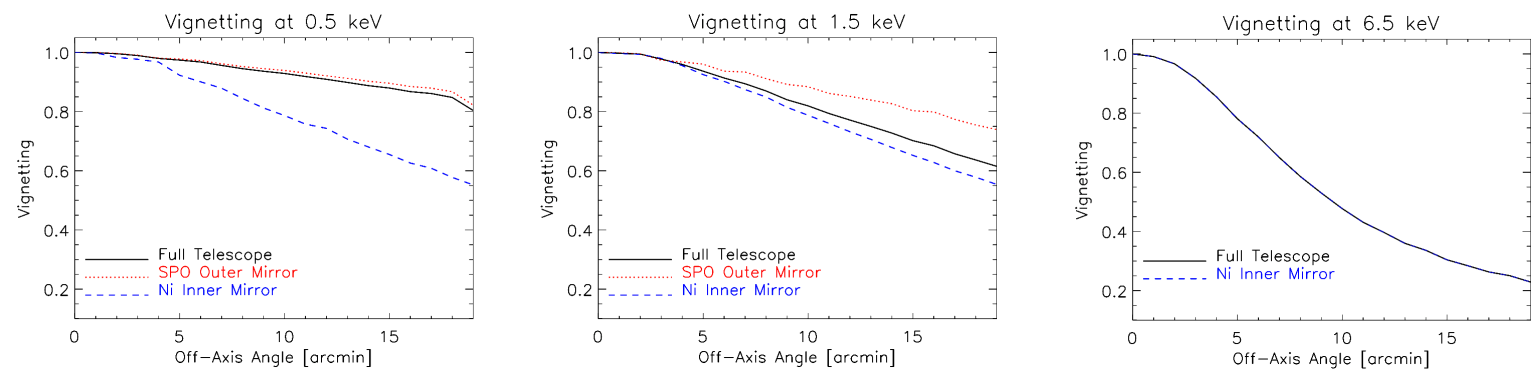

Figure 7. Vignetting vs. off-axis angles at three incident photon energies, namely $0.5,1.5$, and $6.5 \mathrm{keV}$ from left to right panels.

\section{SUMMARY AND CONCLUSIONS}

We have presented the main design characteristics and simulated performances of the optical module of the Cryogenic $\mathrm{X}$-Ray Imaging Spectrometer of the mission ORIGIN, a medium size high-energy mission concept submitted to ESA in response to the Cosmic Vision M3 call issued on July 2010.

In order to meet the scientific requirements of the ORIGIN mission, namely: 1) Energy range 0.2-8 keV; 2) Effective area $>1000$ cm2@1 keV, and >100 cm2@6 keV; 3) FOV > 30'; and 4) Angular Resolution HEW < 30" on axis, and the short focal length constraints deriving from fast re-pointing of transient events, we have proposed a telescope design based on a hybrid mirror technology. The outer part of the mirror module is based on SPO technology and the inner part on standard Ni electroforming technology. This choice reduces the mass since the SPO is very lightweight but still provides the high-energy response with the Ni mirror module filling the region at radius $<30 \mathrm{~cm}$ where the SPO technology is not yet demonstrated.

We have briefly described the mirror technologies that are proposed for the construction of the telescope and have presented the main design parameters. The telescope optic will have a double cone profile for the outer module and Wolter-I profile for the inner module. An optimization study of the reflecting coatings has been performed to obtain both high response in the soft band (Carbon over-coating) and get increased efficiency at the energy range of the Iron K lines (multilayers).

Using a ray-tracing code previously developed for the calibration of Beppo-SAX and SWIFT-XRT, and properly modified to account for the CRIS telescope characteristics, we have simulated the expected performances of the telescope in terms of effective area, energy response, and angular resolution.

The calculated effective area is significantly larger than the requirements at $1 \mathrm{keV}$ and consistent with the requirements at $6.5 \mathrm{keV}$ using Au coating for the shells of the inner mirror module. The use of a more sophisticated ML coating on the outer shells of the inner mirror module can provide a factor two gain in the EA at $6.5 \mathrm{keV}$.

The angular resolution of the telescope has been derived including, beside the intrinsic aberrations of the adopted geometry, an additional error of 16" HEW for the SPO to account for figure errors of the plates in the stacks and mounting tolerances of the MM in the telescope structure according to the present achievements of this technology. The calculated angular resolution on-axis is marginally above the requirements (32" HEW on-axis) due to the error budget of the SPO technology. The angular resolution is not dominated by the conical approximation (22" HEW on-axis), therefore, the expected improvements in the SPO technology will allow an improvement of the angular resolution maintaining the current telescope design.

The current design of the outer mirror module significantly relies on the IXO mirror module development program carried out at ESA. For this reason we quoted in the proposal a Technology Readiness Level (TRL) 4-5 for the outer mirror module. For the inner mirror module based on Ni electroforming technology, the TRL is 9 if we choose the bare Au coating, since the technology has been already successfully used in space in several missions, while it lowers to 5-6 for the ML coating option. 


\section{REFERENCES}

[1]. Smith, S.J., Bandler, S.R., Brekosky, R.P., Brown, A.D., Chervenak, J.A., Eckart, M.E., Figueroa-Feliciano, E., Finkbeiner, F.M., Kelley, R.L., Kilbourne, C.A., Porter, F.S., Sadleir, J.E, "Development of Position-Sensitive Transition-Edge Sensor X-Ray Detectors", IEEE Trans.Appl.Superc., 19, 451-455 (2009).

[2]. M. Bavdaz, A. Peacock, E. Tomaselli et al., "Progress at ESA on high energy optics technologies," in Proc. SPIE vol. 5168, pp. 136-147 (2003).

[3]. M. Beijersbergen, S. Kraft, M. Bavdaz et al., "Development of X-ray pore optics: novel high-resolution silicon millipore optics for XEUS and ultra-low mass glass micropore optics for imaging and timing," in Proc. SPIE, vol. 5539, pp. 104-115 (2004).

[4]. Citterio O., Conti G., Mattaini E., Santambrogio E., and Sacco B., "Optics for X-ray concentrators on board of the Astronomy Satellite SAX", 1986, Proc. SPIE Vol. 587, p. 102-110.

[5]. Aschenbach B., "In-orbit performance of the XMM-Newton X-ray telescopes: images and spectra", 2002, Proc. SPIE Vol. 4496, p.8-22.

[6]. Citterio O., Conconi P., Ghigo M., Loi R., Mazzoleni F., Poretti E., Conti G., Mineo T., Sacco B., Bräuninger H.W., Burkert W.,"X-ray optics for the JET-X experiment aboard the Spectrum X satellite", 1994, Proc. SPIE Vol. 2279, p. 480-492.

[7]. Pareschi G., Citterio O., Ghigo M., Mazzoleni F., Gorenstein P., Romaine S.E., and Parodi G., "Replication by Ni electroforming approach to produce the Con-X/HXT hard x-ray mirrors", 2003, Proc. SPIE Vol. 4851, pp. 528-537.

[8]. M. J. Collon, R. G"unther, M. Ackermann et al., "Stacking of silicon pore optics for IXO," in Optics for EUV, XRay, and Gamma-Ray Astronomy IV, vol. 7437 of Proceedings of SPIE, San Diego, Calif, USA, August 2009.

[9]. M. B. O. Riekerink, B. Lansdorp, L. J. de Vreede et al., "Production of silicon mirror plates," Proc. SPIE, vol. 7437, p. 27 (2009).

[10]. Marcos Bavdaz, Max Collon, Marco Beijersbergen, KotskaWallace, and EricWille, "X-Ray Pore Optics Technologies and Their Application in Space Telescopes", X-Ray Optics and Instrumentation, 2010, Volume 2010, Article ID 295095.

[11]. R. G“"unther, M. Collon, S. Kraft et al., "Production of silicon pore optics,”, Proc. SPIE, vol. 6266 (2006).

[12]. M. J. Collon, R. G"unther, S. Kraft et al., "Silicon pore optics for astrophysical X-ray missions", Proc. SPIE, vol. 6688 (2007).

[13]. M. D. Ackermann, M. J. Collon, R. G"unther et al., "Performance prediction and measurement of silicon pore optics," Proc. SPIE, vol. 7437, (2009).

[14]. M. Bavdaz, PH. Gondoin, K. Wallace et al., "IXO system studies and technology preparation", Proc. SPIE, vol. 7437, (2009).

[15]. M. Bavdaz, E. Wille, K. Wallace et al., "ESA optics technology preparation for IXO", Proc. SPIE, vol. 7732, (2010).

[16]. O. Citterio, G. Bonelli, G. Conti, E. Mattaini, E. Santambrogio, B. Sacco, E. Lanzara, H. Brauninger, and W. Burkert, Optics for the X-ray imaging concentrator aboard the X-ray astronomy satellite SAX, Appl. Opt. 27, 1470 (1988)

[17]. Gondoin, P., Aschenbach, B., Beijersbergen, M. W., et al., Calibration of the first XMM flight mirror module: I image quality. In Proc. SPIE, vol. 3444, p. 278-289 (1998)

[18]. D. N. Burrows, J. E. Hill, J. A., Nousek, A. A. Wells, A. D. Short, R. Willingale, O. Citterio, G. Chincarini, and G.Tagliaferri, Swift X-Ray Telescope, Proc. SPIE, 4140, 64 (2000)

[19]. O. Citterio, M. Ghigo, F. Mazzoleni, G. Pareschi, L. Peverini, Development of soft and hard X-ray optics for astronomy, Proc. SPIE 4138, 43 (2000)

[20]. G. Pareschi et al., Nickel replicated multilayer optics for soft and hard X-ray telescopes, Proc. SPIE 4012, 284 (2000)

[21]. G. Pareschi, O. Citterio, M. Ghigo, F. Mazzoleni, P. Gorenstein, S.E. Romaine, G. Parodi, Replication by Ni electroforming approach to produce the Con-X/HXT hard $x$-ray mirrors, Proc. SPIE 4851, 528 (2003)

[22]. G. Sironi et al., Thin Gold layer in NiCo and Ni electroforming process: optical surface characterization, Proc. SPIE 7732, 77322R (2010)

[23]. D. Vernani et al., Technologies for manufacturing of high angular resolution multilayer coated optics for the New Hard X-ray Mission: a status report II, Proc. SPIE, Vol. 7732, 77323P (2010)

[24]. B. Ramsey et al., The development of hard X-ray optics at MSFC, SPIE Proc., 5168, 129 (2003) 
[25]. S. Romaine et al., Development of a prototype nickel optic for the Constellation-X hard x-ray telescope: III, Proc. SPIE, 5900, 59000S (2005)

[26]. G. Tagliaferri et al., Simbol-X Hard X-ray Focusing Mirrors: Results Obtained During the Phase A Study, AIP Conference Proceedings, CP1126, 35-40 (2009)

[27]. G. Pareschi et al., Design and development of the SIMBOL-X hard X-ray optics, Proc. SPIE, 7011, 70110N (2008)

[28]. S. Basso et al., The optics system of the New Hard X-ray Mission: design and development, Proc. SPIE Vol. 7732, 773218 (2010)

[29]. S. Basso, V. Cotroneo, G. Pareschi, The relation between the weight and the quality image in a telescope, with a particular regard to SIMBOL-X. Mem. S.A.It., Vol. 79, 224-225 (2008)

[30]. G. Conti, E. Mattaini, E. Santambrogio, B. Sacco, G. Cusumano, O. Citterio, H.W. Braeuninger, Burkert W., X-ray characteristics of the Italian X-Ray Astronomy Satellite (SAX) flight mirror units, Proc. SPIE Vol. 2279, p. 101109, (1994).

[31]. G. Cusumano, et al., In-flight calibration of the Swift XRT effective area, AIP Conf. Proc., Vol. 836, p.664-667 (2006).

[32]. E. Perinati, T. Mineo, G. Cusumano, L. Piro, G. Pareschi, M. Barbera, Designing an X-ray baffle for stray light reduction at the focal plane of the Wide Field Imager on-board EDGE, Proc. SPIE Vol.7011, p. 701139-701139-8 (2008).

[33]. G. Cusumano, M. Artale, V. Cotroneo, T. Mineo, G. Pareschi, SIMBOL-X: x-ray baffle for stray-light reduction, Proc. SPIE, vol. 6688, p. 66880C, (2007).

[34]. Barbera M., Mineo T., Perinati E., Schnopper H.W., Taibi A., Thin Plastic Foil X-ray Optics with Spiral Geometry, 2007, Proc. SPIE, Vol. 6688, p. 15-1

[35]. M. Barbera, T. Mineo,; E. Perinati,; H. W. Schnopper, D. Spiga, A. Taibi, Spiral conical approximations to double reflection Wolter optics, 2008, Proc. SPIE, Vol. 7011, pp. 701136-701136-7.

[36]. E. Perinati, T. Mineo, L. Colasanti, S. Lotti, C. Macculi, L. Piro, Estimate of the background for the x-ray microcalorimeter Spectrometer onboard of IXO, Proc. SPIE Vol. 7732, pp. 77323-77323-8 (2010)

[37]. Nartallo, R. E. Daly, H. Evans, P. Nieminen, F. Lei, P. Truscott, Low-angle scattering of protons on the XMMNewton optics and effects on the on-board CCD detectors, IEEE Tran. on Nucl. Sci. 48, 1815 (2001).

[38]. Collon, M. J. et al., Performance of silicon pore optics, Proc. SPIE Vol. 7011, 70111E, (2008)

\section{ACKNOWLEDGEMENTS}

We thank Max Collon and Marcelo Ackermann of Cosine Research (Leiden, NL) for useful suggestions and discussions. We acknowledge partial support by UNIPA, INAF and ASI. SRON is supported financially by NWO, the Netherlands Organization for Scientific Research. 